\title{
Comparison of experimental data on the spin resonance frequency and gap magnitudes in Fe-based superconductors
}

\author{
M.M. Korshunover, ${ }^{a, b}$ V.A. Shestakov ${ }^{b}$, Yu.N. Togushova ${ }^{b}$ \\ ${ }^{a}$ Kirensky Institute of Physics, Federal Research Center KSC SB RAS, 660036 Krasnoyarsk, Russia \\ ${ }^{b}$ Siberian Federal University, Svobodny Prospect 79, 660041 Krasnoyarsk, Russia
}

\begin{abstract}
Here we review experimental data on the peak in inelastic neutron scattering (INS) and superconducting gaps extracted from various experimental techniques. Comparison of energy scales gives the confidence that the observed peak in INS for most materials is the true spin resonance.
\end{abstract}

Keywords: Fe-based superconductors, inelastic neutron scattering, unconventional superconductivity, magnetic mechanism of pairing

PACS: 74.70.Xa, 74.20.Rp, 78.70.Nx, 75.40.Gb

\section{Introduction}

Fe-based superconductors (FeBS) represent a noncuprate class of high- $T_{c}$ systems with the unconventional superconducting state, which origin is still debated. In general, FeBS can be divided into the two subclasses, pnictides and chalcogenides [1]. Different mechanisms of Cooper pairs formation result in the distinct superconducting gap symmetry and structure [2]. In particular, the RPA-SF (random-phase approximation spin fluctuation) approach gives the extended $s$-wave gap that changes sign between hole and electron Fermi surface sheets ( $s_{ \pm}$state) as the main instability for the wide range of doping concentrations $[3,4,5,6,7]$. On the other hand, orbital fluctuations promote the order parameter to have the sign-preserving $s_{++}$symmetry [8]. Thus, probing the gap structure can help in elucidating the underlying mechanism. In this respect, inelastic neutron scattering (INS) is a useful tool since the measured dynamical magnetic susceptibility $\chi(\mathbf{q}, \omega)$ in the superconducting state carries information about the gap structure. For the $s_{ \pm}$state as well as for an extended

Email address: mkor@iph.krasn.ru (M.M. Korshunov) non-uniform $s$-wave gap, nesting wave vector $\mathbf{q}=\mathbf{Q}$ connects Fermi sheets with the different signs of gaps. This fulfills the resonance condition for the interband susceptibility, and the spin resonance peak is formed at a frequency $\omega_{R}[9,10]$ in contrast to the $s_{++}$state where a gradual increase of spin response should take place unless additional scattering mechanisms are assumed $[11,12]$.

\section{Analysis of experimental data}

In Ref. [13], we studied the magnetic response of FeBS with two different superconducting gap scales, $\Delta_{L}>\Delta_{S}$. Spin resonance appears in the $s_{ \pm}$state below the indirect gap scale $\tilde{\Delta}$ that is determined by the sum of gaps on two different Fermi surface sheets connected by the scattering wave vector $\mathbf{Q}$. For the Fermi surface geometry characteristic to the most of FeBS materials, the indirect gap is either $\tilde{\Delta}=\Delta_{L}+\Delta_{S}$ or $\tilde{\Delta}=2 \Delta_{L}$. This gives the simple criterion to determine whether the experimentally observed peak in inelastic neutron scattering is the true spin resonance - if the peak frequency $\omega_{R}$ is less than the indirect gap $\tilde{\Delta}$, then it is the spin resonance and, consequently, the superconducting state has the $s_{ \pm}$gap structure. 
Sometimes it is not always clear experimentally which gaps are connected by the wave vector $\mathbf{Q}$. Even without knowing this exactly, one can draw some conclusions. For example, if one of the gaps is $\Delta_{L}$, then there are three cases possible: (1) $\omega_{R} \leq \Delta_{L}+\Delta_{S}$ and the peak at $\omega_{R}$ is the spin resonance, (2) $\omega_{R} \leq 2 \Delta_{L}$ and the peak is most likely the spin resonance but the definitive conclusion can be drawn only from the calculation of the dynamical spin susceptibility for the particular experimental band structure, and (3) $\omega_{R}>2 \Delta_{L}$ and the peak is definitely not a spin resonance. In the latter case, the peak could be coming from the $s_{++}$state [11, 12], where it forms at frequencies above $2 \Delta$ due to the redistribution of the spectral weight upon entering the superconducting state when a special form of scattering in the normal state is assumed.

Here we combine data on the peak frequency $\omega_{R}$ and maximal and minimal gap sizes $\Delta_{L}$ and $\Delta_{S}$ available in the literature. Results are presented in Table 1 and illustrated in Fig. 1. Unfortunately, for many materials either the INS data or gaps estimations are absent. Latter is shown by question marks in the Table. This gives a whole set of tasks for future experiments. Here are some conclusions, which we can make based on the available data:

1. In electron-doped $\mathrm{BaFe}_{1-x} \mathrm{Co}_{x} \mathrm{As}_{2}$ system, $\mathrm{NaFe}_{1-x} \mathrm{Co}_{x}$ As system, and FeSe, $\omega_{R}<\Delta_{L}+\Delta_{S}$ and, thus the peak in INS is the true spin resonance evidencing sign-changing gap.

2. Some hole doped $\mathrm{Ba}_{1-x} \mathrm{~K}_{x} \mathrm{Fe}_{2} \mathrm{As}_{2}$ materials satisfy $\omega_{R} \leq \Delta_{L}+\Delta_{S}$ condition, and some satisfy $\omega_{R}<$ $2 \Delta_{L}$ condition. Latter comes especially from newer tunneling [27, 31] and Andreev reflection [35] data reveling smaller gap values. The fact that $\omega_{R}<$ $2 \Delta_{L}$ is still consistent with the sign-changing gap, but as we mentioned before, the calculation of the spin response for the particular experimental band structure is required to make a final conclusion.

3. The only case when $\omega_{I N S}>2 \Delta_{L}$ is $\mathrm{FeTe}_{0.5} \mathrm{Se}_{0.5}$. According to our analysis, there should be no signchanging gap structure. But before concluding this since this is the single case only, gap data coming from $\mu$ SR $[48,49]$ should be double checked by independent techniques.

4. Interesting to note, that ARPES in all cases gives gaps values larger than extracted from other techniques. Natural question arise - whether the ARPES overestimates or all other methods underestimates superconducting gaps?

Acknowledgements. We would like to thank H. Kontani, S.A. Kuzmichev, T.E. Kuzmicheva, and V.M.
Pudalov for useful discussions. We acknowledge partial support by RFBR (grant 16-02-00098), and Government Support of the Leading Scientific Schools of the Russian Federation (NSh-7559.2016.2).

[1] See, e.g. M.V. Sadovskii, Physics-Uspekhi 51, 1201 (2008); D.C. Johnston, Advances in Physics 59, 803 (2010); G.R. Stewart, Rev. Mod. Phys. 83, 1589 (2011).

[2] P.J. Hirschfeld, M.M. Korshunov, and I.I. Mazin, Rep. Prog. Phys. 74, 124508 (2011).

[3] I.I. Mazin et al., Phys. Rev. Lett. 101, 057003 (2008).

[4] S. Graser et al., New. J. Phys. 11, 025016 (2009).

[5] K. Kuroki et al., Phys. Rev. Lett. 101, 087004 (2008).

[6] S. Maiti et al., Phys. Rev. B 84, 224505 (2011).

[7] M.M. Korshunov, Physics-Uspekhi 57, 813 (2014).

[8] H. Kontani and S. Onari, Phys. Rev. Lett. 104, 157001 (2010).

[9] M.M. Korshunov and I. Eremin, Phys. Rev. B 78, 140509(R) (2008).

[10] T.A. Maier and D.J. Scalapino, Phys. Rev. B 78, 020514(R) (2008).

[11] S. Onari, H. Kontani, and M. Sato, Phys. Rev. B 81, 060504(R) (2010)

[12] S. Onari and H. Kontani, Phys. Rev. B 84, 144518 (2011).

[13] M.M. Korshunov, V.A. Shestakov, and Yu.N. Togushova, Phys. Rev. B 94, 094517 (2016).

[14] M. Wang et al., Phys. Rev. B 93, 205149 (2016).

[15] D.S. Inosov et al., Nature Physics 6, 178 (2010).

[16] J.T. Park et al., Phys. Rev. B 82, 134503 (2010).

[17] K. Terashima et al., Proc. Nat. Acad. Sci. USA 106, 7330 (2009).

[18] T. Kawahara et al., Physica C 470, S440 (2010).

[19] M. Tortello et al., Phys. Rev. Lett. 105, 237002 (2010).

[20] Y. Yin et al., Phys. Rev. Lett. 102, 097002 (2009).

[21] M.D. Lumsden et al., Phys. Rev. Lett. 102, 107005 (2009).

[22] F. Massee et al., Phys. Rev. B 79, 220517(R) (2009).

[23] S.X. Chi et al., Phys. Rev. Lett. 102, 107006 (2009).

[24] J. Zhao et al., Phys. Rev. Lett. 110, 147003 (2013).

[25] A.D. Christianson et al., Nature 456, 930 (2008).

[26] J.-P. Castellan et al., Phys. Rev. Lett. 107, 177003 (2011).

[27] L. Shan et al., Phys. Rev. Lett. 108, 227002 (2012).

[28] H. Ding et al., EPL 83, 47001 (2008).

[29] L. Wray et al., Phys. Rev. B 78, 184508 (2008).

[30] Y. Zhang et al., Phys. Rev. Lett. 105, 117003 (2010).

[31] T. Shimojima et al., Science 332, 564 (2011).

[32] L. Zhao et al., Chin. Phys. Lett. 25, 4402 (2008).

[33] C. Ren et al., Phys. Rev. Lett. 101, 257006 (2008).

[34] C. Zhang et al., Sci. Rep. 1, 115 (2011).

[35] M. Abdel-Hafiez et al., Phys. Rev. B 90, 054524 (2014).

[36] D.V. Evtushinsky et al., Phys. Rev. B 79, 054517 (2009).

[37] D.V. Evtushinsky et al., New J. Phys. 11, 055069 (2009).

[38] P. Samuely et al., Physica C 469, 507 (2009).

[39] K. Nakayama et al., Phys. Rev. B 83, 020501(R) (2011).

[40] N. Xu et al., Phys. Rev. B 88, 220508(R) (2013).

[41] Y. Zhang et al., Nature Materials 10, 273 (2011).

[42] Q. Wang et al., Nature Materials 15, 159 (2016).

[43] S. Kasahara et al., Proc. Natl. Acad. Sci. USA 111, 16309 (2012).

[44] Y.G. Ponomarev et al., J. Supercond. Nov. Magn. 26, 2867 (2013).

[45] H.A. Mook et al., Phys. Rev. Lett. 104, 187002, (2010).

[46] S.-H. Lee et al., Phys. Rev. B 81, 220502(R) (2010).

[47] J. Wen et al., Phys. Rev. B 81, 100513(R) (2010).

[48] P.K. Biswas et al., Phys. Rev. B 81, 092510 (2010).

[49] M. Bendele et al., Phys. Rev. B 81, 224520 (2010).

[50] C.C. Homes et al., Phys. Rev. B 81, 180508(R) (2010). 
Table 1. Comparison of peak energies in INS and larger and smaller gaps in various FeBS. Data on gap sizes $\Delta_{L}$ and $\Delta_{S}$ extracted from the Andreev reflections data, tunneling spectra and STS, optical spectroscopy, muon spin rotation $(\mu \mathrm{SR})$, the BCS fit of $H_{c 1}(T)$, and angle-resolved photoemission spectroscopy (ARPES) are presented. If the peak frequency and gaps satisfy condition $\omega_{I N S}<\Delta_{L}+\Delta_{S}$, gaps are marked by green color, and if they satisfy condition $\omega_{I N S}<2 \Delta_{L}$, gaps are marked by yellow color. Red color is used in the case of $\omega_{I N S}>2 \Delta_{L}$.

\begin{tabular}{|c|c|c|c|}
\hline Material & $T_{c}(\mathrm{~K})$ & $\omega_{I N S}(\mathrm{meV})$ & $\Delta_{L}, \Delta_{S}(\mathrm{meV})$ \\
\hline $\mathrm{BaFe}_{1.9} \mathrm{Co}_{0.1} \mathrm{As}_{2}$ & 19 & $8.3[14]$ & 5.0, 4.0 (ARPES) [14] \\
\hline $\mathrm{BaFe}_{1.866} \mathrm{Co}_{0.134} \mathrm{As}_{2}$ & 25 & $8.0[14]$ & 6.5, 4.6 (ARPES) [14] \\
\hline $\mathrm{BaFe}_{1.81} \mathrm{Co}_{0.19} \mathrm{As}_{2}$ & 19 & $8.5[14]$ & 5.6, 4.6 (ARPES) [14] \\
\hline $\mathrm{BaFe}_{1.85} \mathrm{Co}_{0.15} \mathrm{As}_{2}$ & 25 & $9.5[15,16]$ & 6.7, 4.5 (ARPES) [17] \\
\hline $\mathrm{BaFe}_{1.85} \mathrm{Co}_{0.15} \mathrm{As}_{2}$ & 25.5 & $\sim 9.5 ?$ & $6.6,5$ (ARPES) [18] \\
\hline $\mathrm{BaFe}_{1.8} \mathrm{Co}_{0.2} \mathrm{As}_{2}$ & 24.5 & $\sim 9.5 ?$ & 9, 4 (Andreev refl.) [19] \\
\hline $\mathrm{BaFe}_{1.85} \mathrm{Co}_{0.15} \mathrm{As}_{2}$ & 25.3 & $?$ & 5.52-6.98 (Tunneling) [20] \\
\hline $\mathrm{BaFe}_{1.84} \mathrm{Co}_{0.16} \mathrm{As}_{2}$ & 22 & $8.6[21]$ & 7 (Tunneling) [22] \\
\hline $\mathrm{BaFe}_{1.9} \mathrm{Ni}_{0.1} \mathrm{As}_{2}$ & 20 & $9.1[23]$ & $?$ \\
\hline $\mathrm{BaFe}_{1.91} \mathrm{Ni}_{0.09} \mathrm{As}_{2}$ & 18 & $6.5-8.7[16]$ & $?$ \\
\hline $\mathrm{Ba}\left(\mathrm{Fe}_{0.65} \mathrm{Ru}_{0.35}\right)_{2} \mathrm{As}_{2}$ & 20 & $8[24]$ & $?$ \\
\hline $\mathrm{Ba}_{0.6} \mathrm{~K}_{0.4} \mathrm{Fe}_{2} \mathrm{As}_{2}$ & 38 & $14[25,26,27]$ & $12.5,5.5$ (ARPES) $[28,29]$ \\
\hline $\mathrm{Ba}_{0.6} \mathrm{~K}_{0.4} \mathrm{Fe}_{2} \mathrm{As}_{2}$ & 38 & $14[25,26,27]$ & 7-11.5, 4-7 (ARPES) [30] \\
\hline $\mathrm{Ba}_{0.6} \mathrm{~K}_{0.4} \mathrm{Fe}_{2} \mathrm{As}_{2}$ & 38 & $14[25,26,27]$ & $8.4,3.2$ (Tunneling) $[27,31]$ \\
\hline $\mathrm{Ba}_{0.6} \mathrm{~K}_{0.4} \mathrm{Fe}_{2} \mathrm{As}_{2}$ & 35 & $14[26]$ & 10-12, 7-8 (ARPES) [32] \\
\hline $\mathrm{Ba}_{0.6} \mathrm{~K}_{0.4} \mathrm{Fe}_{2} \mathrm{As}_{2}$ & 37.5 & $14[26]$ & $8.5-9.3,1.7-2.3\left(H_{c 1}\right)[33]$ \\
\hline $\mathrm{Ba}_{0.67} \mathrm{~K}_{0.33} \mathrm{Fe}_{2} \mathrm{As}_{2}$ & 38 & 15 [34] & $?$ \\
\hline $\mathrm{Ba}_{0.65} \mathrm{~K}_{0.35} \mathrm{Fe}_{2} \mathrm{As}_{2}$ & 34 & $13[26]$ & 7.4-8, 1.4-2 (Andreev spec.) [35] \\
\hline $\mathrm{Ba}_{1-x} \mathrm{~K}_{x} \mathrm{Fe}_{2} \mathrm{As}_{2}$ & 32 & $14[26]$ & 9.2, 1.1 (ARPES) $[36,37]$ \\
\hline $\mathrm{Ba}_{0.55} \mathrm{~K}_{0.45} \mathrm{Fe}_{2} \mathrm{As}_{2}$ & 23 & $?$ & 9.2, 2.7 (Andreev refl.) [38] \\
\hline $\mathrm{Ba}_{0.3} \mathrm{~K}_{0.7} \mathrm{Fe}_{2} \mathrm{As}_{2}$ & 22 & $?$ & 7.9, 4.4 (ARPES) [39] \\
\hline $\mathrm{Ba}_{0.1} \mathrm{~K}_{0.9} \mathrm{Fe}_{2} \mathrm{As}_{2}$ & 9 & $?$ & 2.7-3.6 (ARPES) [40] \\
\hline $\mathrm{K}_{0.8} \mathrm{Fe}_{2} \mathrm{Se}_{2}$ & 31 & $?$ & 10.3 (ARPES) [41] \\
\hline $\mathrm{Cs}_{0.8} \mathrm{Fe}_{2} \mathrm{Se}_{2}$ & 30 & $?$ & 10.3 (ARPES) [41] \\
\hline $\mathrm{FeSe}$ & 8 & $4[42]$ & $2.5,3.5$ (Tunneling) [43] \\
\hline $\mathrm{FeSe}$ & 8 & $4[42]$ & 0.6-1,2.4-3.2 (Andreev refl.) [44] \\
\hline $\mathrm{FeTe}_{0.5} \mathrm{Se}_{0.5}$ & 14 & $6-7[45,46,47]$ & 2.61, 0.51-0.87 ( $\mu \mathrm{SR})[48,49]$ \\
\hline $\mathrm{FeTe}_{0.55} \mathrm{Se}_{0.45}$ & 14 & $?$ & 5.1, 2.5 (Optics) $[50,51]$ \\
\hline $\mathrm{FeTe}_{0.6} \mathrm{Se}_{0.4}$ & 14 & $6.5[53,52]$ & $?$ \\
\hline $\mathrm{Fe}_{1.03} \mathrm{Te}_{0.7} \mathrm{Se}_{0.3}$ & 13 & $?$ & 4 (ARPES) [54] \\
\hline $\mathrm{Tl}_{0.63} \mathrm{~K}_{0.37} \mathrm{Fe}_{1.78} \mathrm{Se}_{2}$ & 29 & $?$ & 8.5 (ARPES) [55] \\
\hline $\mathrm{BaFe}_{2}\left(\mathrm{As}_{0.65} \mathrm{P}_{0.35}\right)_{2}$ & 30 & $12[56,57]$ & $?$ \\
\hline $\mathrm{LaFeAsO}_{0.92} \mathrm{~F}_{0.08}$ & 29 & $13[58]$ & $?$ \\
\hline $\mathrm{LaFeAsO}_{0.943} \mathrm{~F}_{0.057}$ & 25 & $11-12[59]$ & $?$ \\
\hline $\mathrm{LaFeAsO}_{1-x} \mathrm{~F}_{x}$ & 22 & $?$ & 5.4, 1.4 (Andreev refl.) [60] \\
\hline LiFeAs & 18 & $8[61]$ & $5-6,2.8-3.5$ (ARPES) $[62,63,64]$ \\
\hline LiFeAs & 18 & $8[61]$ & $5.4,1.4$ (Andreev refl.) $[65,66]$ \\
\hline LiFeAs & 18 & $8[61]$ & $5.3,2.5$ (Tunneling) $[67,68,69]$ \\
\hline $\mathrm{NaFe}_{0.978} \mathrm{Co}_{0.022} \mathrm{As}$ & 18 & $7.5[70]$ & 2.8 (Optics) [70] \\
\hline $\mathrm{NaFe}_{0.935} \mathrm{Co}_{0.045} \mathrm{As}$ & 18 & $7[71]$ & $6.8,6.5$ (ARPES) [72] \\
\hline $\mathrm{NaFe}_{0.95} \mathrm{Co}_{0.05} \mathrm{As}$ & 18 & $\sim 7 ?$ & $6.8,6.5$ (ARPES) [72] \\
\hline $\mathrm{NdFeAsO}_{0.9} \mathrm{~F}_{0.1}$ & 53 & $?$ & 15 (ARPES) [73] \\
\hline
\end{tabular}




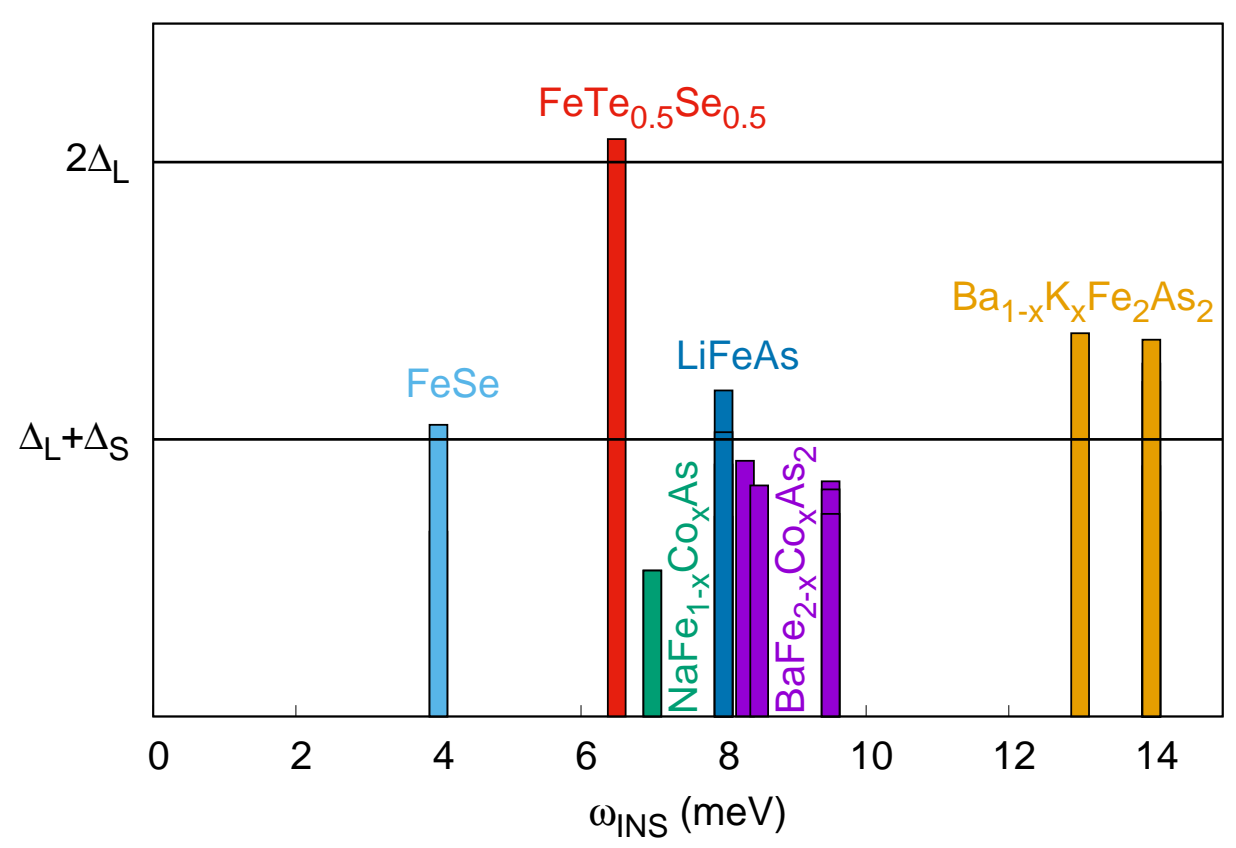

Figure 1. (Color online) Data from Table 1 grouped by materials. Each bar height is determined by $\omega_{I N S} /\left(\Delta_{L}+\Delta_{S}\right)$. If it's below $\Delta_{L}+\Delta_{S}$ boundary, then case (1) is realized; case (2) occurs once it's below $2 \Delta_{L}$ line, and situation (3) corresponds to the intersection of the $2 \Delta_{L}$ limit.

[51] C.C. Homes et al., J. Phys. Chem. Sol. 72, 505 (2011).

[52] D.N. Argyriou et al., Phys. Rev. B 81, 220503(R) (2010).

[53] Y.M. Qiu et al., Phys. Rev. Lett. 103, 067008 (2009).

[54] K. Nakayama et al., Phys. Rev. Lett. 105, 197001 (2010).

[55] X.-P. Wang et al., EPL 93, 57001 (2011).

[56] M. Ishikado et al., Physica C 471, 643 (2011).

[57] M. Ishikado et al., Phys. Rev. B 84, 144517 (2011).

[58] S. Shamoto et al., Phys. Rev. B 82, 172508 (2010).

[59] S. Wakimoto et al., J. Phys. Soc. Jpn. 79, 074715 (2010).

[60] S.A. Kuzmichev et al., J. Supercond. Nov. Magn. 29, 1111 (2016).

[61] A.E. Taylor et al., Phys. Rev. B 83, 220514(R) (2011).

[62] S.V. Borisenko et al., Phys. Rev. Lett. 105, 067002 (2010).

[63] S.V. Borisenko et al., Symmetry 4, 251 (2012).

[64] K. Umezawa et al., Phys. Rev. Lett. 108, 037002 (2012).

[65] S.A. Kuzmichev et al., JETP Lett. 95, 537 (2012).

[66] S.A. Kuzmichev et al., JETP Lett. 98, 722 (2013).

[67] S. Chi et al., Phys. Rev. Lett. 109, 087002 (2012).

[68] T. Hanaguri et al., Phys. Rev. B 85, 214505 (2012).

[69] P.K. Nag et al., Sci. Rep. 6, 27926 (2016).

[70] A. Charnukha et al., Sci. Rep. 6, 18620 (2016).

[71] C. Zhang et al., Phys. Rev. B 88, 064504 (2013).

[72] Z.-H. Liu et al., Phys. Rev. B 84, 064519 (2011).

[73] T. Kondo et al., Phys. Rev. Lett. 101, 147003 (2008). 